\title{
Síndrome da Apneia Obstrutiva do Sono e Doenças Cardiovasculares
}

\author{
Obstructive Sleep Apnea and Cardiovascular Diseases
}

\author{
Daniella D. Azzari Campostrini ${ }^{1}$ Lucila B. Fernandes do Prado ${ }^{2}$, \\ Gilmar Fernandes do Prado ${ }^{3}$
}

\section{RESUMO}

Introduçáo. A Síndrome da Apneia Obstrutiva do Sono (SAOS) é caracterizada por obstrução parcial ou completa da via aérea superior, de forma intermitente e recorrente durante o sono. As principais manifestações durante o sono incluem roncos, pausas respiratórias, fragmentação do sono, levando a prejuízos diurnos incapacitantes, como sonolência excessiva diurna, alteraçóes cognitivas e cefaléia matinal. Além das manifestaçóes respiratórias clássicas, resulta em alterações metabólicas e hemodinâmicas que predispóem ao desenvolvimento e agravamento de doenças cardiovasculares, tais como hipertensão arterial sistêmica, insuficiência cardíaca congestiva, doença arterial coronariana e arritmias. Objetivo. Abordar aspectos clínicos, fisiopatológicos e diagnósticos da SAOS, bem como seus efeitos deletérios no sistema cardiovascular. Método. Revisão da literatura acerca da SAOS e suas consequências cardiovasculares através de artigos selecionados no PubMed. Conclusáo. A apneia obstrutiva do sono e as doenças cardiovasculares têm origem multifatorial e alta prevalência na população mundial. Considerando que as doenças cardiovasculares são a maior causa atual de morbidade e mortalidade no mundo, a determinação de fatores de risco possíveis de controle e tratamento, como a SAOS, torna-se fundamental para prevençáo e tratamento das doenças cardiovasculares.

Unitermos. Síndrome da Apneia Obstrutiva do Sono, Doenças Cardiovasculares, Hipertensão, insuficiência Cardíaca Congestiva, Doença da Artéria Coronariana, Arritmias Cardíacas.

Citaçáo. Campostrini DDA, Prado LBF, Prado GF. Síndrome da Apneia Obstrutiva do Sono e Doenças Cardiovasculares.

\begin{abstract}
Introduction. Obstructive Sleep Apnea Syndrome (OSAS) is characterized by partial or complete obstruction of the upper airway, intermittently and recurrently during sleep. The main manifestations during sleep include snoring, pauses in breathing, sleep fragmentation, resulting in excessive daytime sleepiness, cognitive dysfunction and morning headaches. Besides the classic respiratory symptoms, OSAS results in metabolic and hemodynamic alterations that predispose to the development and worsening of cardiovascular diseases such as hypertension, congestive heart failure, coronary artery disease and arrhythmias. Objective. Reporting clinical, pathophysiological and diagnostic aspects of OSAS and its deleterious effects on the cardiovascular system. Method. Review of literature about OSAS and its cardiovascular consequences through selected articles in PubMed. Conclusion. Obstructive sleep apnea and cardiovascular diseases have multifactorial origin and high prevalence in the global population. Because cardiovascular diseases are the main cause of the current morbidity and mortality worldwide, determining possible treatable risk factors, such as OSAS, it is fundamental to prevention and treatment of cardiovascular diseases.
\end{abstract}

Keywords. Obstructive Sleep Apnea, Cardiovascular Disease, Hypertension, Heart Failure, Coronary Artery Disease, Cardiac Arrhythmias.

Citation. Campostrini DDA, Prado LBF, Prado GF. Obstructive Sleep Apnea and Cardiovascular Diseases.

\footnotetext{
Trabalho realizado no Setor de Neurofisiologia Clínica do Departamento de Neurologia e Neurocirurgia, Escola Paulista de Medicina - EPM, Universidade Federal de Sáo Paulo - UNIFESP, Sáo Paulo-SP, Brasil.

1.Neurologista, residente de Neurofisiologia Clínica da EPM-UNIFESP, São Paulo-SP, Brasil.

2.Neurofisiologista com atuação em Medicina do Sono, Doutora, Setor de Neuro-Sono da Disciplina de Neurologia da EPM-UNIFESP, São Paulo-SP, Brasil.

3.Neurologista, Doutor, Professor Livre Docente, Setor de Neuro-Sono da Disciplina de Neurologia da EPM-UNIFESP, São Paulo-SP, Brasil.
}

Endereço para correspondência: Dra Lucila Prado Rua Cláudio Rossi, 394 CEP 01547-000, São Paulo-SP, Brasil. Fone/fax: 11 5081-6629 E-mail: neuro.sono.unifesp@gmail.com 


\section{INTRODUÇÃO}

A Síndrome da Apneia Obstrutiva do Sono (SAOS) é uma doença sistêmica comum, porém subdiagnosticada.Descrita em 2 a $4 \%$ da população mundial ${ }^{1}$.

Caracteriza-se por obstrução parcial ou completa da via aérea superior (VAS), de forma intermitente e recorrente durante o sono, resultando em períodos de apneia, hipóxia, esforços ventilatórios, seguidos de despertar transitório e desobstrução da via aérea, com restauração do fluxo aéreo ${ }^{2}$.

Os episódios de obstrução da VAS ocorrem em todos os estágios do sono, especialmente no sono REM (rapid eye movement - movimentos oculares rápidos) e no estágio N2 do sono não REM. Durante o sono REM as apneias tendem a ser mais longas e a dessaturação da oxi-hemoglobina mais acentuada, devido principalmente a atonia nesse estágio do sono ${ }^{2}$.

As principais manifestações durante o sono incluem roncos, pausas respiratórias, fragmentação do sono, levando a prejuízos diurnos incapacitantes, como sonolência excessiva diurna, alteraçóes cognitivas e cefaléia matinal.

Embora a SAOS se apresente predominantemente com sintomas respiratórios, as consequências cardiovasculares são de suma importância. A SAOS coexiste com a doença cardiovascular, podendo estar envolvida no seu início, progressão e resistência às estratégias terapêuticas convencionais ${ }^{3}$. Dentre as doenças cardiovasculares que estão associadas com a SAOS, destacam-se a hipertensão arterial sistêmica, insuficiência cardíaca congestiva, doença arterial coronariana e arritmias.

As doenças cardiovasculares são a maior causa atual de morbidade e mortalidade no mundo ${ }^{4}$. Na cidade de São Paulo, as doenças cardiovasculares associadas à aterosclerose também representam a principal causa de óbito semelhante aos países desenvolvidos ${ }^{5,6}$. Esses dados reforçam a necessidade de novos trabalhos para esclarecer os diversos fatores risco para as doenças cardiovasculares, para que sejam estabelecidas melhores estratégias para combatê-los.

O objetivo do presente estudo foi abordar a SAOS como modulador e fator de risco para doenças cardiovasculares, através de uma revisão da literatura. Serão abordados aspectos clínicos, diagnósticos e fisiopatológicos da SAOS, bem como seus efeitos deletérios no sistema cardiovascular.

\section{DIAGNÓSTICO}

O diagnóstico de SAOS é baseado na história clínica, no exame físico e na polissonografia.

A história clínica deve ser detalhada, sendo altamente aconselhável a presença do parceiro ou familiar próximo. A simples observação de roncos, gasping e apneia noturna, associados à sonolência diurna, proporciona alta suspeição para apneia obstrutiva do sono ${ }^{7}$.

Os sinais e sintomas são divididos em noturnos e diurnos, incluindo ronco, apneia testemunhada, engasgos noturnos, noctúria, sono frangmentado, sonolência excessiva diurna, cefaleia matinal, além de prejuízos na concentração, atenção e memória.

O ronco é o sintoma mais frequente na apneia obstrutiva do sono, ocorre em até $95 \%$ dos pacientes. Apenas $6 \%$ dos pacientes com apneia não referem ronco $^{8-10}$.

Durante o exame físico é fundamental avaliação do peso, altura, circunferência do pescoço e cálculo do Índice de Massa Corporal (IMC). A circunferência do pescoço tem se correlacionado melhor com SAOS quando comparada ao IMC. A obtenção de uma circunferência cervical superior a $40 \mathrm{~cm}$ deve levar a suspeita de desordens respiratórias relacionadas ao sono ${ }^{11}$.

O objetivo do exame da VAS e da cavidade nasal é identificar estruturas ou anormalidades com potencial de obstruir e aumentar a resistência ao fluxo aéreo durante o sono. Também deve ser avaliada a estrutura do esqueleto craniofacial de forma individualizada, já que anormalidades como retrognatia e micrognatia estão associadas a um maior risco de colapso da VAS ${ }^{12}$.

O estudo polissonográfico de noite inteira realizado em laboratório é o principal método diagnóstico para os distúrbios respiratórios do sono. A American Academy of Sleep Medicine (AASM) recomenda a realização da PSG em duas noites. Na primeira realiza-se o diagnóstico e na outra se titula o $\mathrm{CPAP}^{13}$.

A PSG fornece dados importantes tanto para o diagnóstico quanto para caracterizar os pacientes em diferentes grupos de gravidade. $\mathrm{O}$ índice mais utilizado é o índice apneia/hipopneia (IAH) que mensura os períodos de apneia e hipopneia por hora de sono. 
Define-se apneia quando ocorre redução do fluxo aéreo $\geq 90 \%$ ou ausência do fluxo aéreo devido ao completo colapso da VAS por pelo menos 10 segundos durante o sono, na presença de esforços ventilatórios ativos que se refletem nos movimentos toracoabdominais ${ }^{13}$ (Figura 1).

Caracteriza-se hipopneia quando há obstrução parcial ao fluxo aéreo, por pelo menos 10 segundos, com redução $\geq 30 \%$ do fluxo aéreo oronasal, associada à queda da saturação da oxi-hemoglobina $\geq 4 \%$, ou redução $\geq$ $50 \%$ do fluxo associado a queda na saturação $\geq 3 \%$ ou critérios eletroencefalográficos de microdespertares durante o sono ${ }^{13}$ (Figura 2 e 3 ).

Baseado na frequência de eventos respiratórios durante o sono (apneia, hipopneia e esforços respiratórios associados ao despertar) a SAOS é classificada como leve (IAH entre 5 e 15 eventos/hora), moderada (IAH entre 15 e 30 eventos/hora) ou grave (IAH > 30 eventos/hora), permitindo-se dimensionar a gravidade dos sintomas e as complicaçóes que podem ocorrer a longo prazo, além de possibilitar o direcionamento do tratamento.

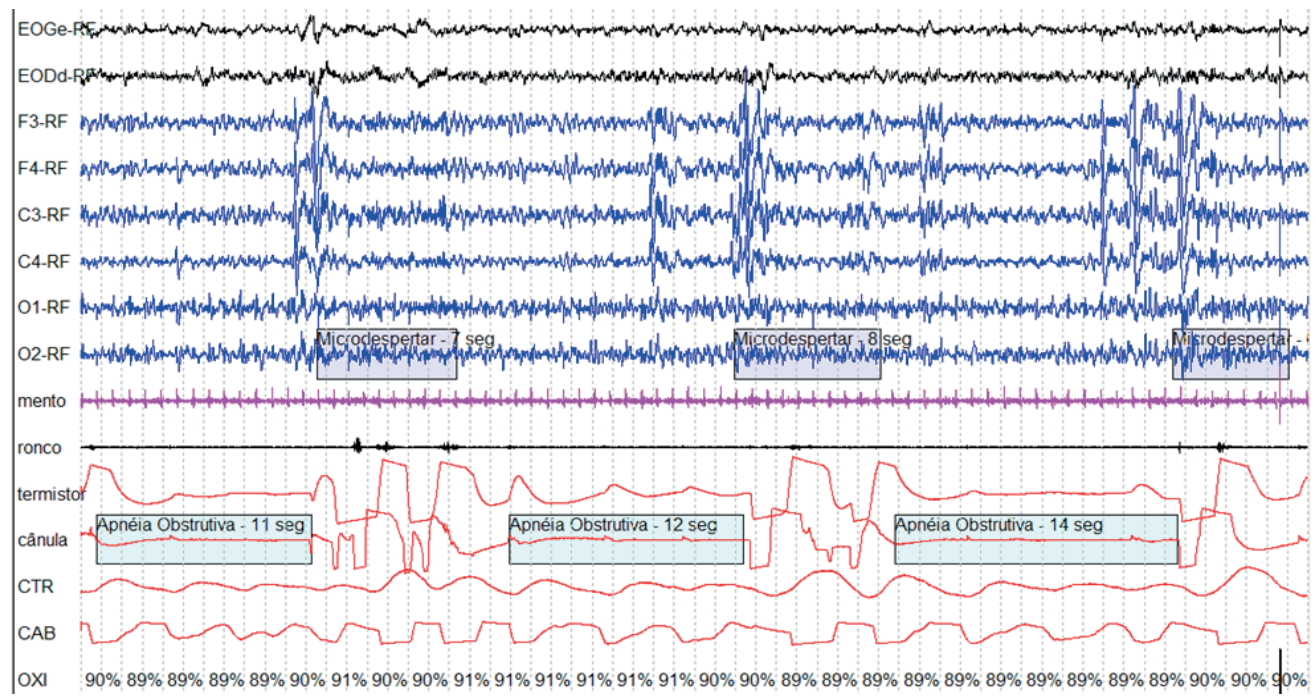

Figura 1. Exame de polissonografia demonstrando episódios de apneia obstrutiva. (Fonte: Laboratório Neuro-Sono - EPM/UNIFESP).

EOGG

Figura 2. Exame de polissonografia demonstrando hipopneia. Destaque para a queda do fluxo de oxigênio (em rosa) e dessaturação (em verde claro). (Fonte: Laboratório Neuro-Sono - EPM/UNIFESP). 




Figura 3. Exame de polissonografia demonstrando hipopneia. Destaque para a queda do fluxo de oxigênio (em rosa), dessaturação (em verde claro) e para o microdespertar (em vermelho). (Fonte: Laboratório Neuro-Sono - EPM/UNIFESP).

\section{FISIOPATOLOGIA}

A fisiopatologia da SAOS é complexa e variável entre os pacientes. Vários são os mecanismos que levam ao colapso da VAS e que, por conseguinte, desencadeiam distúrbios respiratórios intermitentes.

Assim, existem forças que tendem ao colapso da faringe, as quais se dividem em internas (pressão negativa durante a inspiração) e externas (acúmulo de gordura e alteraçóes craniofaciais). Em contrapartida, existem forças que neutralizam essa tendência, como a tensão dos músculos da faringe, o controle central do tônus da faringe e a tração traqueal.

Dentre os possíveis mecanismos que contribuem para obstrução da VAS e surgimento da SAOS, podemos citar alteraçôes craniofaciais, influências genéticas, o efeito da posição supina e da gravidade durante o sono, o uso de álcool e sedativos antes de dormir e a obesidade.

Pacientes obesos e com síndrome metabólica possuem uma incidência elevada de SAOS e esta aumenta de forma significativa a incidência de aterosclerose e risco cardiovascular ${ }^{14,15}$.

Enquanto a obesidade aumenta o risco de SAOS, a própria apneia do sono pode predispor ao ganho de peso. Portanto, descreve-se um ciclo vicioso em que a obesidade predispóe ao aparecimento da SAOS, e essa se perpetua através da sua influência negativa sobre o peso corporal.
Assim, podemos dizer que a SAOS é uma doença multifatorial, em que a associação dos fatores descritos contribui de forma sinérgica para a obstruçáo da VAS.

\section{CONSEQUENNCIAS CARDIOVASCULARES}

A obstruçáo da VAS durante o sono leva a alterações do fluxo oronasal e aumento do esforço respiratório, com consequente queda da saturação da oxi-hemoglobina (ou seja, hipoxemia) e hipercapnia. Esse processo leva à ativação do sistema nervoso simpático, com aumento da frequência cardíaca, da resistência vascular e da pressão arterial, culminando num microdespertar que restabelece a ventilação normal por contrair a musculatura da faringe. Esses eventos alternam-se várias vezes durante a noite.

Durante cada episódio de apneia/hipopneia obstrutiva a inspiração forçada contra a via aérea ocluída é acompanhada de pressão negativa no espaço pleural. $\mathrm{Na}$ medida em que a apneia se prolonga, acentuam-se a hipoxemia e a hipercapnia, levando à vasoconstrição pulmonar, com o desenvolvimento de hipertensão pulmonar transitória.

A estimulação do sistema nervoso simpático pode provocar um aumento significativo da pressão arterial durante o sono, mesmo em indivíduos com pressão arterial normal durante a vigília ${ }^{16}$.

O fenômeno de hipoxemia e reoxigenação recor- 
rentes, repetido por inúmeras vezes durante a noite, leva ao desenvolvimento de estresse oxidativo com formação de radicas livres, que é atualmente considerado um fator importante para o desenvolvimento das doenças cardiovasculares observadas nesses pacientes com SAOS ${ }^{17,18}$.

A hipóxia leva ao aumento das citocinas inflamatórias, como o fator de necrose tumoral alfa (TNF- $\alpha$ ) e a interleucina 6 (IL-6), que estão envolvidas na regulaçâo fisiológica do sono e encontram-se anormalmente elevadas em pacientes apneicos quando comparados com indivíduos normais e com pacientes portadores de obesidade ${ }^{19}$. Também ocorre aumento de vasoconstritores, como a endotelina-1, com redução de fatores vasodilatadores, como óxido nítrico. Esses fatores levam a disfunção endotelial, a qual contribui para piorar a aterosclerose, trombose vascular e disfunção de ventrículo esquerdo. A disfunção endotelial está presente em várias doenças cardiovasculares, como hipertensão arterial e doença coronariana.

Esses dados nos mostram que os episódios de apneia e hipopneia, com hipoxemia e reoxigenação recorrentes durante o sono, desencadeiam diversos efeitos sistêmicos, o que sugere fortemente que a SAOS seja uma doença sistêmica e não uma anormalidade local.

\section{Hipertensão Arterial Sistêmica}

A SAOS e a hipertensão arterial sistêmica (HAS) são doenças altamente prevalentes na população e coexistem num mesmo paciente de forma significativa. A relação entre essas duas doenças se deve, em grande parte, ao fato de a SAOS funcionar como um modelo de ativação simpática persistente, com diminuição na sensibilidade de barorreceptores, hiperresponsividade vascular e alteração no metabolismo do sal e da água, contribuindo para o aumento da pressão arterial.

Vários estudos tem demonstrado a associação entre SAOS e HAS, estimando-se que cerca de 50\% a $70 \%$ dos pacientes com SAOS sejam hipertensos e que 30\% dos hipertensos tenham SAOS ${ }^{20,21}$. Indivíduos com diagnóstico de HAS podem apresentar cerca de 2 vezes mais chances de ter SAOS que os indivíduos sem $\mathrm{HAS}^{22}$.

Atualmente está bem estabelecido o papel da SAOS como fator de risco independente para HAS. O Wisconsin Sleep Cohort Study realizou uma análise multivariada com ajuste para outros fatores de risco para doença cardiovas- cular, como tabagismo, obesidade, alcoolismo e pressão arterial sistêmica. Após 4 anos de seguimento, mostrou-se um associação causal entre presença de SAOS na avaliação inicial e surgimento de HAS no segmento, independentemente de outros fatores de risco, com risco 3 vezes maior para desenvolvimento de HAS. Além disso, constatou que essa associação era dependente da gravidade da SAOS ao mostrar que para cada aumento do IAH $(0,1$ a 4,9, 5 a 14,9, e > 15 eventos/hora de sono) atribuiu-se um crescente odds ratio (OR) para desenvolver hipertensão arterial $(1,42,2,03 \text {, e 2,89, respectivamente })^{23}$.

A SAOS tem sido reconhecida ainda como a principal causa secundária de hipertensão arterial resistente pelas diretrizes europeias e americanas e pela VI Diretriz Brasileira de Hipertensão Arterial ${ }^{24}$. Estudos investigam o papel do hiperaldosteronismo como importante ponte dessa relação, principalmente na SAOS grave, com resultados promissores do uso de antagonista da aldosterona nessa população ${ }^{25,26}$.

Pacientes com SAOS tem grande variabilidade da pressão arterial durante a noite, podendo não apresentar o descenso noturno observado em pessoas normais, levando a um valor médio noturno elevado, mesmo nas situaçôes em que se observam níveis pressóricos normais durante o dia. Isso pode ocorrer principalmente devido ativaçáo simpática mantida durante o sono de pacientes apneicos. O descenso atenuado $(<10 \%)$ ou ausente é preditor independente de risco cardiovascular, mais importante até que a pressão arterial diurna ${ }^{21,27}$.

Vários estudos abordam o benefício do tratamento da SAOS para redução dos níveis tensionais, especialmente devido ao controle da atividade simpática associada ao tratamento efetivo com CPAP, sendo proporcional ao seu tempo de uso noturno ${ }^{28}$. Estudos mostram que a terapia regular com CPAP por no mínimo 5 horas por noite em pacientes com SAOS resulta em benefício na redução dos níveis tensionais tanto sistólicos como diastólicos. Resultados de 12 meta-análises de ensaios randomizados CPAP-placebo apontam para redução de 1,69 mmHg na média dos níveis tensionais aferidos em monitorização ambulatorial de 24 horas $^{29}$.

Os preditores de melhor resposta encontrados são: SAOS grave, maiores níveis tensionais e maior adesão ao $\mathrm{CPAP}^{30}$. Entretanto, nem todos os estudos confirmam 
esses benefícios, mas seus resultados devem ser interpretados com cautela, uma vez que esses estudos incluem populações heterogêneas, apresentam variaçôes na definição de HAS e na classificação da gravidade da SAOS, bem como no tempo de uso do CPAP e nos métodos de monitorização da pressão $\operatorname{arterial}^{31}$.

\section{Insuficiência Cardíaca}

A prevalência de SAOS entre pacientes portadores de insuficiência cardíaca varia de $10 \%$ a $35 \%^{32,33}$ e mais da metade dos pacientes com SAOS apresenta disfunção diastólica que tende a melhorar com o uso do CPAP ${ }^{34}$.

O estudo Sleep Heart Health mostrou que pacientes com SAOS (com IAH > 11/hora) tem um risco ${ }^{2,38}$ vezes maior para desenvolver insuficiência cardíaca, independentemente de outros fatores de risco ${ }^{35}$.

Dentre os possíveis mecanismos para o aumento do risco de insuficiência cardíaca em pacientes com SAOS, destaca-se o aumento da ativação simpática noturna nesses pacientes, com consequente elevação dos níveis tensionais. Esse aumento da pressão arterial é o mecanismo diretamente responsável pela disfunção sistólica do ventrículo esquerdo. $\mathrm{O}$ aumento dos níveis de citocinas, catecolaminas, endotelina e fatores de crescimento envolvidos na fisiopatologia da SAOS também contribui para o surgimento de hipertrofia ventricular esquerda, independentemente da presença de hipertensão arterial ${ }^{36}$. Além disso, a hipóxia noturna determina isquemia e apoptose dos miócitos, sendo fator de risco independente para prejuízo do relaxamento ventricular durante a diástole, o que causa maior estresse na parede ventricular, favorecendo a dilatação dessa câmara e piorando o prognóstico a longo prazo ${ }^{37}$.

Além dos mecanismos citados, devemos destacar as mudanças repetitivas nas pressóes intratorácicas que acompanham os episódios de apneia e hipopneia durante o sono. Ao se exercer pressão intratorácica inspiratória negativa contra a faringe ocluída, ocorre aumento da pressão transmural do ventrículo esquerdo e, consequentemente, de sua pós-carga. Esse mecanismo também aumenta o retorno venoso, incrementando a pré-carga do ventrículo direito. Enquanto isso, a hipóxia provoca vasoconstrição dos vasos pulmonares, levando a hipertensão pulmonar e ao aumento da pós-carga do ventrículo direito. A combi- nação de maior pós-carga e menor pré-carga do ventrículo esquerdo leva a progressiva reduçáo do volume sistólico e do débito cardíaco. Esses mecanismos são recorrentes durante o sono e causam significativa sobrecarga pressórica no ventrículo esquerdo, além de necrose e apoptose de miócitos, responsáveis pelo aumento do risco de infarto agudo do miocárdio, disfunção sistólica, arritmias, e remodelamento cardíaco ${ }^{38}$.

Por outro lado, acredita-se que a insuficiência cardíaca também possa colaborar no desenvolvimento da SAOS, uma vez que causa diminuição do tônus muscular da VAS e acúmulo de fluidos em partes moles da região cervical, facilitando a tendência ao colapso da VAS durante o sono.

Os efeitos a longo prazo do uso do CPAP nas funçôes sistólica e diastólica permanecem incertos. Não está claro se o tratamento com CPAP pode retardar a progressão da insuficiência cardíaca ou melhorar a função cardiovascular ${ }^{39-41}$.

Em relação à hipertensão pulmonar, vários estudos apontam para o benefício do tratamento da SAOS pela diminuição da hipóxia e sua ação na vasorreatividade pulmonar e na melhora da disfunção endotelial ${ }^{12,43}$. Avaliação de 12 semanas de terapia efetiva com CPAP demonstrou redução de 4,9 + 3,9mmHg (IC 95\% 3,04$6,67 \mathrm{mmHg}$ ) $[15,1+11,2 \%$ (IC 95\% 10,1-20,3\%) $]$ nos níveis da pressão sistólica da artéria pulmonar, passando de 28,9 + 8,6 $\mathrm{mmHg}$ para $24+5,8 \mathrm{mmHg}(\mathrm{p}<0,0001)$. Assim, admite-se que o tratamento adequado em longo prazo com o CPAP poderia evitar o desenvolvimento de alteraçôes estruturais pulmonares e do ventrículo direito, mudando o prognóstico desses pacientes ${ }^{43}$.

\section{Arritmias Cardíacas}

Mais de 50\% dos portadores de SAOS apresentam arritmias noturnas e a frequência das arritmias aumenta com o IAH e a gravidade da hipoxemia ${ }^{44}$. O risco de episódio de fibrilação atrial (FA) ou de taquicardia ventricular não-sustentada 90 minutos após um episódio de apneia/hipopneia é 18 vezes maior em relação aos períodos de respiração normal.44 A prevalência de taquicardia ventricular não sustentada é maior em indivíduos com SAOS em relação aos indivíduos normais ${ }^{45}$.

As arritmias mais comuns durante o sono são: ta- 
quicardia ventricular não-sustentada, taquicardia sinusal, bloqueio atrioventricular de $2^{\circ}$ grau e extrassístoles frequentes $^{46}$.

Em 2006, Mehraet al..$^{45}$ compararam 228 pacientes com distúrbios respiratórios do sono e 338 pacientes sem. Os pacientes com distúrbios graves apresentaram 2 a 4 vezes maior risco de arritmias complexas noturnas, tais como FA e taquicardia ventricular não-sustentada, mesmo após ajuste para idade, sexo, IMC e presença de doença arterial coronariana.

Vários estudos tentam explicar os mecanismos pelos quais a SAOS aumenta o risco de arritmias cardíacas. As oscilaçôes entre o sistema simpático e parassimpático que ocorrem durante o sono nos pacientes com SAOS levam a um meio perfeito para o surgimento das arritmias. O predomínio do tônus parassimpático favorece a ocorrência das bradiarritmias, enquanto o predomínio do tônus simpático, leva as taquiarritmias atriais e ventriculares ${ }^{3}$.

A hipóxia, na ausência de fluxo aéreo, causa um estímulo dos quimiorreceptores carotídeos com consequente bradicardia devido estimulação vagal eferente. Já durante a presença do fluxo aéreo, como ocorre no período pós apneia, ocorre estiramento do pulmão levando à inibição do parassimpático com instalaçâo do predomínio do simpático ${ }^{3}$. Portanto, durante os episódios de apneia pode ocorrer diminuição da frequência cardíaca seguido de aumento da mesma no período pós apneia, o que configura a Síndrome Bradicardia-taquicardia em pacientes com SAOS.

Muitos estudos demonstram que a SAOS atua como fator de risco independente para o desenvolvimento de FA, sendo que a SAOS moderada a grave aumenta em até 4 vezes o risco dessa arritmia ${ }^{45}$. Aproximadamente $24 \%$ dos pacientes com FA possuem SAOS e 32\% a $49 \%$ dos pacientes com SAOS grave podem desenvolver FA no futuro ${ }^{47,48}$. A ocorrência e recorrência da FA apresentada por estes pacientes provavelmente deve-se ao ciclo de hipoxemia intermitente, ativação simpática e alteraçốes abruptas da pressão arterial ${ }^{49}$. Esses fatores levam ao remodelamento atrial que ocorre devido ao persistente aumento do tônus simpático e variaçôes da pressão e da distensão atrial, fator importante para o aparecimento de arritmias atriais ${ }^{1}$.
Flutuações crônicas da frequência cardíaca, pressão arterial e intratorácica podem levar ao remodelamento miocárdico, que culmina com o desenvolvimento de hipertrofia do ventrículo esquerdo e direito seguida de disfunção sistólica e diastólica. Esses mecanismos estão envolvidos com aumento do risco de arritmias ventriculares e morte súbita.

Gami et al. demonstraram que os portadores de SAOS apresentam maior risco de morte súbita à noite, sugerindo associação entre os dois problemas. $\mathrm{O}$ mecanismo exato que explica o elo entre SAOS e morte súbita ainda não está bem esclarecido ${ }^{50}$.

Alguns prováveis mecanismos tentam explicar a causa de morte súbita nesses pacientes com SAOS. O primeiro deles é que a hipoxemia aumenta a atividade do sistema nervoso autônomo, o estado pró-coagulante e pró-inflamatório, facilitando a ocorrência de isquemia miocárdica. Outro mecanismo é a parada sinusal e o bloqueio atrioventricular, que ocorrem nos pacientes durante a predominância do tônus parassimpático. Por último, o aumento das arritmias ventriculares nesses pacientes, como resultado da isquemia miocárdica e da ativação do sistema nervoso autônomo simpático, influencia diretamente no evento súbito que culmina em morte ${ }^{1}$.

Diversos estudos apontam para a ação benéfica do CPAP nos eventos obstrutivos, com consequente redução da pressão intratorácica, da hipóxia, da acidose e dos despertares, e impacto na redução das arritmias cardíacas, principalmente a $\mathrm{FA}^{51,52}$.

Estudos mostram que pacientes com SAOS apresentam risco $25 \%$ maior de recorrência de FA após ablação quando comparados a pacientes sem $\mathrm{SAOS}^{53}$. Os pacientes com SAOS apresentam menor sucesso na ablação e mais complicaçóes relacionadas ao procedimento. Os pacientes em tratamento com CPAP tiveram menores taxas de recorrência de $\mathrm{FA}^{54}$.

\section{Doença Arterial Coronariana}

Vários estudos tentam definir a relação causal entre SAOS e doença arterial coronariana (DAC). Estima-se que a prevalência de DAC em pacientes com SAOS esteja em torno de $25 \%$, sendo esta porcentagem mais alta em pacientes com SAOS moderada a grave. Já a prevalência de SAOS em pacientes com DAC é estimada em 30\% ${ }^{35}$. 
Conforme discutido previamente, pacientes com SAOS apresentam aumento da atividade nervosa simpática periférica tanto durante o sono como na vigília, podendo contribuir dessa forma para os eventos coronários agudos nas primeiras horas da manhā ${ }^{55}$.

Além do aumento do tônus simpático, a SAOS leva a alteraçôes metabólicas, endoteliais e neuro-humorais desencadeadas, basicamente, por períodos de hipóxia e reoxigenação recorrentes durante o sono. Com isso ocorrem aumentos da pressão arterial e da frequência cardíaca, disfunção endotelial e instalação de estado pró-inflamatório, favorecendo, assim, a maior aterogênese, a qual é o principal fator associado às doenças cardiovasculares, especialmente ao desenvolvimento da $\mathrm{DAC}^{56}$.

O estado pró-inflamatório pode estar representado pela maior expressão de diversos mediadores, como TNF- $\alpha$, IL-6 e proteína C-reativa ${ }^{56}$. E a disfunção endotelial ocorre devido diversos fatores, incluindo a maior expressão de moléculas de adesão, como a molécula de adesão intercelular 1 (ICAM-1), a molécula de adesão à célula vascular 1 (VCAM-1) e a E-selectina. Bem como, maior liberação de substâncias vasoativas, como a endotelina que pode contribuir para o desenvolvimento de vasoconstrição sustentada em pacientes SAOS, aumentando o risco de doenças cardiovasculares. Existem evidências de que a SAOS é um fator de risco independente para disfunção endotelial ${ }^{57}$.

\section{TRATAMENTO}

O tratamento da SAOS deve ser multidisciplinar, com o objetivo de atingir o controle dos sintomas e reduzir o risco cardiovascular.

Inicialmente, todos os pacientes portadores de distúrbio do sono devem se submeter à mudança comportamental, que inclui: boa higiene do sono; descontinuar o uso de drogas e/ou fármacos, como álcool, benzodiazepínicos, opioides e barbitúricos; cessar o tabagismo e identificar e tratar processos alérgicos e infecciosos da VAS; perda de peso; evitar posiçáo supina durante o sono e manter a cama com inclinação de 30-60 graus; tratar refluxo gastroesofágico e hipotireoidismo; resolver a obstrução nasal decorrente de anormalidades anatômicas ou congestão nasal.

O tratamento de escolha para SAOS moderada a grave é o uso do CPAP (Continuous Positive Airway Pressure $)^{56,58-60}$. Considerado método com maiores benefícios, tanto na apneia quanto nos desfechos cardiovasculares ${ }^{61}$. Aplicado preferencialmente por máscara nasal, o CPAP proporciona pressão positiva durante todo o ciclo respiratório, que mantém a permeabilidade da VAS durante a inspiração e a expiração. Dessa forma, impede o colapso das paredes faringeanas, a ocorrência de apneias e hipopneias e a vibração de estruturas moles da faringe, evitando assim o ronco.

O valor da pressão que será utilizada no CPAP é definido pelo uso da PSG para sua titulação. Durante a PSG, inicialmente aplica-se uma pressão fisiológica (4 $\mathrm{cmH}_{2} \mathrm{O}$ ) e um tempo de rampa (tempo que o aparelho leva para alcançar uma pressão alvo). Esta rampa dependerá do tempo de latência do sono de cada paciente, tentado assim, só expor o paciente a pressóes altas e incômodas quando estiver dormindo.

A pressão ideal é aquela que proporciona a supressão dos eventos obstrutivos e do ronco, a recuperação da saturação de oxigênio e a abolição dos despertares noturnos em quaisquer posiçóes.

A indicação de CPAP para o tratamento da SAOS tem como desafio a adesão do paciente, principalmente naqueles pouco sintomáticos. Para melhor adesão, os efeitos colaterais devem ser resolvidos, como por exemplo, congestão nasal, coriza, rinorréia, lesão cutânea na ponte do nariz, aerofagia com distensão gástrica. Os critérios que definem a adesão são o uso mínimo de 4 horas por noite em $70 \%$ das noites em período de 30 dias consecutivos ${ }^{62}$.

Os aparelhos intraorais são indicados pela AASM para pacientes com SAOS leve a moderada e para pacientes com SAOS grave que não tiveram sucesso ou náo toleraram o $\mathrm{CPAP}^{63}$. Podem ser divididos em dispositivos de avanço temporário da mandíbula e de tração da musculatura lingual, que aumentam a passagem de ar na VAS. Contraindicados em caso de dor, disfunçóes temporomandibulares graves não tratadas e doença periodontal ativa. Os principais efeitos colaterais são desconforto mandibular, sensibilidade dental e sialorreia.

A indicação de cirurgia para o tratamento da SAOS ainda continua controversa ${ }^{64}$. Pode ser considerada como terapia adjunta ao CPAP para pacientes com apneia obs- 
trutiva do sono moderada e grave com obstrução anatômica que possa ser corrigida cirurgicamente (como hipertrofia tonsilar com obstrução da VAS). Também pode ser usada como segunda opçáo para um grupo restrito de pacientes, dentre os quais aqueles que náo apresentaram sucesso no tratamento com CPAP ou aparelhos intraorais. São várias as alternativas cirúrgicas disponíveis na atualidade: cirurgia nasal, cirurgia orofaríngea, redução da base da língua e cirurgia maxilomandibular. A cirurgia nasal tem impacto no controle do ronco, mas sem benefício para tratamento da $\mathrm{SAOS}^{65}$. Já a cirurgia orofaríngea é indicada na presença de hipertrofia das tonsilas palatinas. Ambas são consideradas na presença do aumento da resistência ao fluxo aéreo, com o intuito de otimizar o uso de aparelhos de pressão na via aérea. Outras cirurgias tem sua indicação restrita atualmente e estão reservadas para casos graves e refratários ao tratamento convencional.

Apesar das divergências de resultados nos estudos que avaliam o uso do CPAP e benefícios cardiovasculares, já está bem estabelecido o benefício do uso do CPAP no tratamento da SAOS, especialmente a SAOS moderada a grave, levando a diminuição dos eventos obstrutivos, melhora do padrão respiratório durante o sono, instituindo sono reparador, com diminuição da sonolência diurna e melhora da qualidade de vida dos pacientes. Além disso, o uso do CPAP é fundamental para atenuar ou reverter as complicaçóes cardiovasculares da SAOS, contribuindo para a redução das pressóes arteriais noturnas e diurnas, redução da incidência de arritmias cardíacas e do risco de morte por evento cardiovascular agudo $28,34,66,67$.

\section{CONCLUSÃO}

A apneia obstrutiva do sono e as doenças cardiovasculares têm origem multifatorial e alta prevalência na população mundial.

Diversos estudos demonstram a relação da apneia obstrutiva do sono com doenças cardiovasculares e morte súbita. A SAOS é uma doença sistêmica e é considerada fator de risco independente para hipertensão arterial, insuficiência cardíaca, doença arterial coronária e arritmias.

Considerando que as doenças cardiovasculares são a maior causa atual de morbidade e mortalidade no mundo, a determinação de fatores de risco possíveis de con- trole e tratamento, como a SAOS, torna-se fundamental para prevenção e tratamento das doenças cardiovasculares.

Apesar disso, o diagnóstico e tratamento da SAOS ainda não recebem a devida importância na prática clínica. Os distúrbios do sono, apesar de altamente prevalentes, são subdiagnosticados e consequentemente não tratados. Isso ocorre em parte pela falta de investigação na rotina da atenção primária e pela falta de conhecimento sobre a importância do tema pelos profissionais da saúde.

Conclui-se, portanto, que o adequado diagnóstico e tratamento da SAOS se faz necessário quando há uma busca constante por melhora da qualidade de vida e sobrevida da população.

\section{REFERÊNCIAS}

1.Hersi AS. Obstructive sleep apnea and cardiac arrhythmias. Ann Thorac Med 2010;5:10-7.

http://dx.doi.org/10.4103/1817-1737.58954

2.Kryger MH, Roth T, Dement WC (eds.). Principles of Sleep Medicine. St. Louis: Elsevier Saunders, 5th edition, 2011, 1928p.

3.Chan KH, Wilcox I. Obstructive sleep apnea: novel trigger and potential therapeutic target for cardiac arrhythmias. Expert Rev Cardiovasc Ther 2010;8:981-94

http://dx.doi.org/10.1586/erc. 10.80

4.Murray CJL, Lopez AD. The global burden of disease: a comprehensive assessment of mortality and disability from disease, injuries and risk factors in 1990 and projected to 2020. Harvard: Harvard Univeresity Press, 1996, 43p. 5.Lolio CA, Laurenti R. Evolução da mortalidade por doença isquêmica do coração no município de Sáo Paulo, 1970 a 1981. Arq Bras Cardiol 1986;46:153-6.

6.Uemura K, Pisa Z. Recent trends in cardiovascular disease mortality in 27 industrialized countries. Wld Hlth Start Quart 1971;38:1617-25.

7.Kingshott RN, Sime PJ, Engleman HM, Douglas NJ. Self assessment of daytime sleepiness: patient versus partner. Thorax 1995;50:994-5.

http://dx.doi.org/10.1136/thx.50.9.994

8.Gottieb DJ, Yao Q, Redline S, Ali T, Mahowald MW. Does snoring predicte sleepiness independently of apnea and hypopnea frequency? Am J Respir Crit Care Med 2000;162:1512-7.

http://dx.doi.org/10.1164/ajrccm.162.4.9911073

9.Flemons WW, Whitelaw WA, Brant R, Remmers JE. Likelihood ratios for a sleep apnea clinical prediction rule. Am J Respir Crit Care Med 1994;150:1279-85.

http://dx.doi.org/10.1164/ajrccm.150.5.7952553

10.Viner S, Szalai JP, Hoffstein V. Are history and physical examination a good screening test for sleep apnea? Ann Intern Med 1991;115:356-9.

http://dx.doi.org/10.7326/0003-4819-115-5-356

11.Phillips BA, Kryger MH. Management of Obstructive Sleep Apnea-Hy- 
popnea Syndrome. In: Kryger MH, Roth T, Dement WC (eds.). Principles of Sleep Medicine. St. Louis: Elsevier Saunders, 5th edition, 2011, p.827-37. http://dx.doi.org/10.1016/B978-1-4160-6645-3.00110-9

http://dx.doi.org/10.1016/B978-1-4160-6645-3.00134-1

12.Jamieson A GC, Partinen M, Quera-Salva MA. Obstructive sleep apneic patients have craniomandibular abnormalities. Sleep 1986; 9:469-77.

13.Iber C, Israel SA, Chesson AL, Quan SF. The AASM manual for the scoring of sleep and associated events: Rules, Terminology, and Technical specifications. Westchester: American Academy of Sleep Medicine, 2007, 59p.

14.Drager LF, Bortolotto LA, Maki-Nunes C, Trombetta IC, Alves MJ, Fraga RF, et al. The incremental role of obstructive sleep apnoea on markers of atherosclerosis in patients with metabolic syndrome. Atherosclerosis 2010;208:490-5.

http://dx.doi.org/10.1016/j.atherosclerosis.2009.08.016

15.Drager LF, Krieger EM, Lorenzi-Filho G. Sympathetic Activity, Heart Failure, Obesity, and Metabolic Syndrome: Is There Any Role for Obstructive Sleep Apnea? Hypertension 2007;49:e38.

http://dx.doi.org/10.1161/HYPERTENSIONAHA.107.090274

16.Strohl KP, Novak RD, Singer W, Cahan C, Boehm KD, Denko CW, et al. Insulin levels, blood pressure and sleep apnea. Sleep 1994;17:614-8.

17.McCord JM. Oxygen-derived free radicals in post-20. ischemic tissue injury. N Engl J Med 1985;312:159-63.

http://dx.doi.org/10.1056/NEJM198501173120305

18. Findley LJ, Boykin M, Fallon T, Belardinelli L. Plasma adenosine and hypoxemia in patients with sleep apnea. J Appl Physiol 1988;64:556-61.

19.Vgontzas AN, Papanicolaou DA, Bixler EO. Elevation of plasma cytokines in disorders of excessive daytime sleepiness: role of sleep disturbance and obesity. J Clin Endocrinol Metab 1997;82:1313-6.

http://dx.doi.org/10.1210/jcem.82.5.3950

http://dx.doi.org/10.1210/jc.82.5.1313

20.Baguette JP, Narkiewicz K, Mallion JM. Update on Hypertension Management: obstructive sleep apnea and hypertension. J Hypertens 2006;24:205-8.

http://dx.doi.org/10.1097/01.hjh.0000198039.39504.63

21.Calhoun DA, Harding SM. SLeep and hypertension. Chest 2010;138:43443.

http://dx.doi.org/10.1378/chest.09-2954

22.Asha'ari ZA, Hasmoni MH, Ab Rahman J, Yusof RA, Ahmad RA. The association between sleep apnea and young adults with hypertension. Laryngoscope 2012;122:2337-42.

http://dx.doi.org/10.1002/lary.23379

23.Peppard PE, Young T, Palta M, Skatrud J. Prospective study of the association between sleep-disordered breathing and hypertension. N Engl J Med 2000;342:1378-84.

http://dx.doi.org/10.1056/NEJM200005113421901

24.Sociedade Brasileira de Nefrologia. VI Diretrizes Brasileiras de Hipertensão. Arq Bras Cardiol 2010; 95(1 supl.1):1-51.

25.Pimenta E, Stowasser M, Gordon RD, Harding SM, Batlouni M, Zhang

B, et al. Increased dietary sodium is related to severity of obstructive sleep apnea in patients with resistant hypertension and hyperaldosteronism. Chest 2013;143:978-83.

http://dx.doi.org/10.1378/chest.12-0802

26.Gonzaga CC, Gaddam KK, Ahmed MI, Pimenta E, Thomas SJ, Harding $S M$, et al. Severity of obstructive sleep apnea is related to aldosterone status in subjects with resistant hypertension. J Clin Sleep Med 2010;6:363-8.

27.Narkiewicz K, Somers VK. The sympathetic nervous system and obstructive sleep apnea: implications for hypertension. J Hypertens 1997;15:1613-9. http://dx.doi.org/10.1097/00004872-199715120-00062

28. Waradekar NV, Sinoway LI, Zwillich CW, Leuenberger UA. Influence of treatment on muscle sympathetic nerve activity in sleep apnea. Am J Respir Crit Care Med 1996;153:1333-8.

http://dx.doi.org/10.1164/ajrccm.153.4.8616563

29. Haentjens P, Van Meerhaeghe A, Moscariello A, De Weerdt S, Poppe K, Dupont A, et al. The impact of continuous positive airway pressure on blood pressure in patients with obstructive sleep apnea syndrome: evidence from a meta-analysis of placebo-controlled randomized trials. Arch Intern Med 2007; 167:757-64.

http://dx.doi.org/10.1001/archinte.167.8.757

30.Dudenbostel T, Calhoun DA. Resistant hypertension, obstructive sleep apnoea and aldosterone. J Hum Hypertens 2012;26:281-7.

http://dx.doi.org/10.1038/jhh.2011.47

31.Rao M, Rajda G, Uppuluri S, Beck GR, Liu L, Bisognano JD. The role of continuous positive airway pressure in the treatment of hypertension in patients with obstructive sleep apnea-hypoapnea syndrome: a review of randomized trials. Rev Recent Clin Trials 2010;5:35-42.

http://dx.doi.org/10.2174/157488710790820526

32. Wang H, Parker JD, Newton GE, Floras JS, Mak S, Chiu KL, et al. Influence of obstructive sleep apnea on mortality in patients with heart failure. J Am Coll Cardiol 2007;49:1625-31.

http://dx.doi.org/10.1016/j.jacc.2006.12.046

33.Javaheri S, Parker TJ, Liming JD, Corbett WS, Nishiyama H, Wexler L, et al. Sleep apnea in 81 ambulatory male patients with stable heart failure. Types and their prevalences, consequences, and presentations. Circulation 1998;97:2154-9.

http://dx.doi.org/10.1161/01.CIR.97.21.2154

34.Sin DD, Fitzgerald F, Parker JD, Newton G, Floras JS, Bradley TD. Risk factors for central and obstructive sleep apnea in 450 men and women with congestive heart failure. Am J Respir Crit Care Med 1999;160:1101-6.

http://dx.doi.org/10.1164/ajrccm.160.4.9903020

35.Shahar E, Whitney CW, Redline S, Lee ET, Newman AB, Javier Nieto F, et al. Seep-disordered breathing and cardiovascular disease: cross-sectional results of the Sleep Heart Health Study. Am J Respir Crit Care Med 2001;163:19-25. http://dx.doi.org/10.1164/ajrccm.163.1.2001008

36. Otto ME, Belohlavek M, Romero-Corral A, Gami AS, Gilman G, Svatikova $A$, et al. Comparison of cardiac structural and functional changes in obese otherwise healthy adults with versus without obstructive sleep apnea. Am J Cardiol 2007;99:1298-302.

http://dx.doi.org/10.1016/j.amjcard.2006.12.052

37.Fung JW, Li TS, Choy DK, Yip GW, Ko FW, Sanderson JE, et al. Severe obstructive sleep apnea is associated with left ventricular diastolic dysfunction. Chest 2002;121:422-9.

http://dx.doi.org/10.1378/chest.121.2.422

38. Floras JS. Clinical aspects of sympathetic activation and parasympathetic withdrawal in heart failure. J Am Coll Cardiol 1993;22(4 Suppl A):72A-84.

http://dx.doi.org/10.1016/0735-1097(93)90466-E

39. Roebuck T, Solin P, Kaye DM, Bergin P, Bailey M, Naughton MT. Increased long-term mortality in heart failure due to sleep apnoea is not yet proven. Eur Respir J 2004;23:735-40.

http://dx.doi.org/10.1183/09031936.04.00060404

40. Wexler L, Javaheri S. Sleep apnea is linked to heart failure, but does treatment improve outcome? Cleve Clin J Med 2005;72:929-36.

http://dx.doi.org/10.3949/ccjm.72.10.929

41.Mansfield DR, Gollogly NC, Kaye DM, Richardson M, Bergin P, Naughton MT. Controlled trial of continuous positive airway pressure in obstructive sleep apnea and heart failure. Am J Respir Crit Care Med 2004;169:361-6. http://dx.doi.org/10.1164/rccm.200306-752OC

42.Sajkov D, Wang T, Saunders NA, Bune AJ, McEvoy RD. Continuous posi- 
tive airway pressure treatment improves pulmonary hemodynamics in patients with obstructive sleep apnea. Am J Respir Crit Care Med 2002;165:152-8. http://dx.doi.org/10.1164/ajrccm.165.2.2010092

43.Arias MA, Garcia-Rio F, Alonso-Fernandez A, Martinez I, Villamor J. Pulmonary hypertension in obstructive sleep apnoea: effects of continuous positive airway pressure: a randomized, controlled cross-over study. Eur Heart J 2006;27:1106-13.

http://dx.doi.org/10.1093/eurheartj/ehi807

44.Monahan K, Storfer-Isser A, Mehra R, Shahar E, Mittleman M, Rottman J, et al. Triggering of nocturnal arrhythmias by sleep-disordered breathing events. J Am Coll Cardiol 2009;54:1797-804.

http://dx.doi.org/10.1016/j.jacc.2009.06.038

45.Mehra R, Benjamin EJ, Shahar E, Gottlieb DJ, Nawabit R, Kirchner HL, et al. Association of nocturnal arrhythmias with sleep-disordered breathing: The Sleep Heart Health Study. Am J Respir Crit Care Med 2006;173:910-6. http://dx.doi.org/10.1164/rccm.200509-1442OC

46.Miller WP. Cardiac arrhythmias and conduction disturbances in the sleep apnea syndrome. Prevalence and significance. Am J Med 1982;73:317-21.

http://dx.doi.org/10.1016/0002-9343(82)90716-1

47.Gami AS, Pressman G, Caples SM, Kanagala R, Gard JJ, Davison DE, et al. Association of atrial fibrillation and obstructive sleep apnea. Circulation 2004;110:364-7.

http://dx.doi.org/10.1161/01.CIR.0000136587.68725.8E

48.Wang TJ, Parise H, Levy D, D'Agostino RB, Wolf PA, Vasan RS, et al. Obesity and the risk of new-onset atrial fibrillation. JAMA 2004;292:2471-7. http://dx.doi.org/10.1001/jama.292.20.2471

49.Haggsträm FM ZE, Fam CF. Apnéia obstrutiva do sono e alteraçôes cardiovasculares. Scientia Medica 2009;19:122-8.

50.Gami AS, Howard DE, Olson EJ, Somers VK. Day-night pattern of sudden death in obstructive sleep apnea. N Engl J Med 2005;352:1206-14.

http://dx.doi.org/10.1056/NEJMoa041832

51.Harbison J, O’Reilly P, McNicholas WT. Cardiac rhythm disturbances in the obstructive sleep apnea syndrome: effects of nasal continuous positive airway pressure therapy. Chest 2000;118:591-5.

http://dx.doi.org/10.1378/chest.118.3.591

52.Abe H, Takahashi M, Yaegashi H, Eda S, Tsunemoto H, Kamikozawa M, et al. Efficacy of continuous positive airway pressure on arrhythmias in obstructive sleep apnea patients. Heart Vessels 2010;25:63-9.

http://dx.doi.org/10.1007/s00380-009-1164-z

53.Ng CY, Liu T, Shehata M, Stevens S, Chugh SS, Wang X. Meta-analysis of obstructive sleep apnea as predictor of atrial fibrillation recurrence after catheter ablation. Am J Cardiol 2011;108:47-51.

http://dx.doi.org/10.1016/j.amjcard.2011.02.343

54.Patel D, Mohanty P, Di Biase L, Shaheen M, Lewis WR, Quan K, et al. Safety and efficacy of pulmonary vein antral isolation in patients with obstructive sleep apnea: the impact of continuous positive airway pressure. Circ Arrhythm Electrophysiol 2010;3:445-51.

http://dx.doi.org/10.1161/CIRCEP.109.858381

55.Carlson JT, Hedner J, Elam M, Ejnell H, Sellgren J, Wallin BG. Augmented resting sympathetic activity in awake patients with obstructive sleep apnea. Chest 1993;103:1763-8.

http://dx.doi.org/10.1378/chest.103.6.1763
56.Somers VK, White DP, Amin R, Abraham WT, Costa F, Culebras A, et al. Sleep apnea and cardiovascular disease: an American Heart Association/ american College Of Cardiology Foundation Scientific Statement from the American Heart Association Council for High Blood Pressure Research Professional Education Committee, Council on Clinical Cardiology, Stroke Council, and Council On Cardiovascular Nursing. In collaboration with the National Heart, Lung, and Blood Institute National Center on Sleep Disorders Research (National Institutes of Health). Circulation 2008;118:1080-111.

http://dx.doi.org/10.1161/CIRCULATIONAHA.107.189420

57.Kato M, Roberts-Thomson P, Phillips BG, Haynes WG, Winnicki M, Accurso $\mathrm{V}$, et al. Impairment of endothelium-dependent vasodilatation of resistence vassels in patients with obstructive sleep apnea. Circulation 2000;102:207-10.

http://dx.doi.org/10.1161/01.CIR.102.21.2607

58.Epstein LJ, Kristo D, Strollo PJ, Friedman N, Malhotra A, Patil SP, et al. Clinical guideline for the evaluation, management and long-term care of obstructive sleep apnea in adults. J Clin Sleep Med 2009;5:263-76.

59.Rosenthal L, Massie CA, Dolan DC, Loomas B, Kram J, Hart RW. A multicenter, prospective study of a novel nasal EPAP device in the treatment of obstructive sleep apnea: efficacy and 30-day adherence. J Clin Sleep Med 2009;5:532-7.

60.Vennelle M, White S, Riha RL, Mackay TW, Engleman HM, Douglas NJ. Randomized controlled trial of variable-pressure versus fixed-pressure continuous positive airway pressure (CPAP) treatment for patients with obstructive sleep apnea/hypopnea syndrome (OSAHS). Sleep 2010;33:267-71.

61.McNicholas WT. Cardiovascular outcomes of CPAP therapy in obstructive sleep apnea syndrome. Am J Physiol Regul Integr Comp Physiol 2007;293:1666-70.

http://dx.doi.org/10.1152/ajpregu.00401.2007

62.Aloia MS, Knoepke CE, Lee-Chiong T. The new local coverage determination criteria for adherence to positive airway pressure treatment: testing the limits? Chest 2010;138:875-9.

http://dx.doi.org/10.1378/chest.09-2237

63. Hoffstein V. Review of oral appliances for treatment of sleep-disordered breathing. Sleep Breath 2007;11:1-22.

http://dx.doi.org/10.1007/s11325-006-0084-8

64.Elshaug AG, Moss JR, Southcott AM, Hiller JE. Redefining success in airway surgery for obstructive sleep apnea: a meta analysis and synthesis of the evidence. Sleep 2007;30:461-7.

65.Li HY, Wang PC, Chen YP, Lee LA, Fang TJ, Lin HC. Critical appraisal and meta-analysis of nasal surgery for obstructive sleep apnea. Am J Rhinol Allergy 2011;25:45-9.

http://dx.doi.org/10.2500/ajra.2011.25.3558

66.Peker Y, Carlson J, Hedner J. Increased incidence of coronary artery disease in sleep apnoea: a long-term follow-up. Eur Resp J 2006;28:596-602.

http://dx.doi.org/10.1183/09031936.06.00107805

67.Haentjens P, Van Meerhaeghe A, Moscariello A, De Weerdt S, Poppe K, Dupont A, et al. The impact of continuous positive airway pressure on blood pressure in patients with obstructive sleep apnea syndrome: evidence from a meta-analysis of placebo-controlled randomized trials. Arch Intern Med 2007;167:757-64.

http://dx.doi.org/10.1001/archinte.167.8.757 